provide the invaluable time dimension missing from the subject matter of evolutionary geneticists and ecologists. We might even learn something, furthermore, not just about phyletic lineages but about the evolution of certain communities or ecosystems. Partly as a reaction to what they regard as the somewhat arid scholasticism of a subject devoted more to classification than problem solving, but more positively because they have become excited by new biological concepts and wish to apply them to fossils, a number of younger researchers would now prefer to call themselves palaeobiologists.

The term was used explicitly three years ago in a well-received book edited by T. J. M. Schopf and entitled Models in Paleobiology, in which a number of authors devoted themselves to setting up and testing models at a variety of levels ranging from individual growth to ecosystems. Schopf is now the joint editor, with his Chicago colleague R. G. Johnson, of a new journal called Paleobiology, published by the American Paleontological Society-the first number appeared in March this year. The editors hope to publish original contributions dealing with any aspect of biological palaeontology, including morphology, biogeochemistry, populations, faunal provinces, communities and ecosystems. The emphasis is on biological processes and patterns including speciation, extinction, development of individuals and colonies, natural selection, evolution and patterns of variation, abundance and distribution in space and time.

How does the first number of the journal measure up to this ambitious programme? Five of the articles are concerned with aspects of functional morphology. Gould and Katz give a computer simulation of the spirally arranged facets of a Silurian receptaculitid and discuss the significance of the geometrical insights thereby obtained. Hopson argues persuasively that the prominent cranial crests of hadrosaurian dinosaurs were sexual display structures and Stanley describes experiments undertaken to throw light on what controls the shape of burrowing bivalves. Two other short articles are concerned with the musculature of brachiopods and crinoids.

The remaining four articles deal with more general matters. Schopf, Raup, Gould and Simberloff continue their computer simulation of phylogeny and argue that fossils of more complex morphology may only appear to have evolved more rapidly, and more controversially that rate of morphological change might bear little or no relation to the underlying genomic evolution. With reference to the evolutionary history of the arthropods, Flessa, Powers and Cisne challenge the widely held assumption that generalised organisms have persisted longer than specialised ones. Raup writes a methodological essay on taxonomic survivorship curves and Levinton and Bambach compare a Silurian with a modern bivalve community. The quality of the papers, in terms of data, thought and presentation, is almost uniformly high.

All the authors of the first issue of Paleobiology and the whole editorial board are American. This reflects the fact that the revitalisation of an old discipline has taken place almost entirely in America, probably chiefly because of the flexible teaching system which will allow a student interested in fossils to take biology courses and equip himself for this new area of research.

\section{Are X-ray stars and pulsars related?}

by John Gribbin

THE juxtaposition of two papers in Nature last week emphasises just how close astronomers may be to producing a unified model of two of the most intriguing discoveries of recent years: $\mathrm{X}$-ray stars and pulsars. According to Tademaru and Harrison (254, 676; 1975) pulsars may be accelerated to high velocities by asymmetric emission of low frequency electromagnetic radiation. This is a welcome suggestion for the theorists, since although the theories require that pulsars are formed in supernova explosions, and supernovae are thought only to occur in binary systems, only one pulsar out of more than 100 discovered is known to be in a binary system. Clearly, for the theories to hold up the pulsars must have some means of escape from the binaries in which they are born, and the Tademaru-Harrison hypothesis is one intriguing possibility.

Clark, Parkinson and Caswell, on the other hand, have been studying Cir X-1, an X-ray source which may well be both a binary and a remnant left over from a supernova explosion (254, 674; 1975). They suggest that $\mathrm{Cir} X-1$ is a runaway from the supernova remnant $\mathrm{G} 321-0.3$, and that the X-ray source is in a highly elliptical orbit around a companion. Most models of X-ray stars require the presence of a companion, from which matter falls on to the X-ray star itself to produce $\mathrm{X}$-rays by conversion of gravitational energy into radiation. So it seems plausible that they may be produced in supernova explosions which have not unbound the parent binary system.

Cir X-1, however, is almost an intermediate case. If the model of Clark et al. is correct, then the ellipticity of the system now seen is so great that the mass transfer, X-ray mechanism switches off for a time around apastron, when the two components are furthest apart, thus mimicking an eclipse. It is very tempting to see an overall pattern emerging from the observations of $\mathrm{X}$-ray stars and pulsars, in which some systems remain tightly bound after the supernova event, producing 'standard' X-ray sources, others become runaway pairs in which the system remains bound but highly elliptical, producing unusual $X$-ray stars such as Cir X-1, and in still others the neutron star escapes completely, running away on its own to be seen as a pulsar.

There is one further, interesting implication of these ideas, and that concerns the nature of the star left behind when a pulsar does run away from such a binary system. As the companion must have been involved in mass exchange during the lifetime of the binary system, it may well seem to be highly evolved for its mass; in addition, of course, it is likely to have been somewhat disturbed by the supernova event itself. One consequence of the Tademaru-Harrison model, however, is that there is no need to suggest that if the pulsar accelerates away from the sight of the explosion in one direction then the companion must have moved away in the opposite direction, as there would be an energy mechanism for moving the pulsar independently of its erstwhile companion. So a search for odd, highly evolved stars near the centres of known supernova remnants might not prove entirely fruitless, even if there is already good reason to associate a known runaway pulsar with the particular supernova in question.

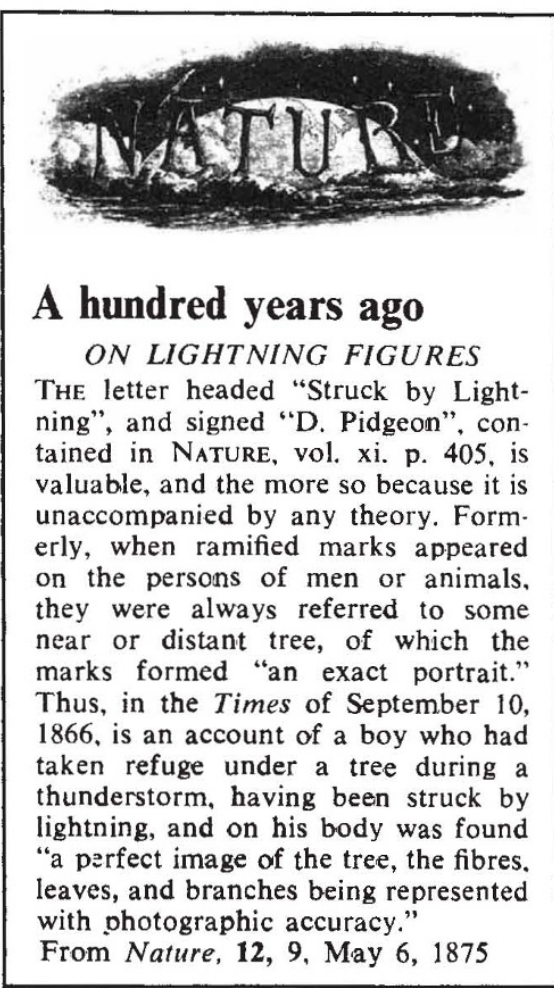

